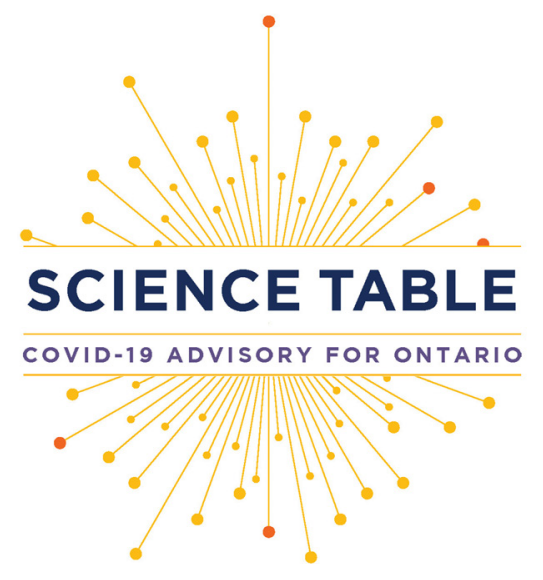

Version: 1.0

Published: February 10, 2022

Citation: Jüni P, Baert S, Corbeil A, et al. Use of rapid antigen tests during the omicron wave. Science Briefs of the Ontario COVID-19 Science Advisory Table. 2022;3(56). https:// doi.org/10.47326/ocsat.2022.03.56.1.0

Author Affiliations: The affiliations of the members of the Ontario COVID-19 Science Advisory Table can be found at https:// covid19-sciencetable.ca/.

Declarations of Interest: The declarations of interest of the members of the Ontario COVID-19 Science Advisory Table, its Working Groups, or its partners can be found at https:// covid19-sciencetable.ca/. The declarations of interest of external authors can be found under additional resources at https://doi. org/10.47326/ocsat.2022.03.56.1.0

About Us: The Ontario COVID-19 Science Advisory Table is a group of scientific experts and health system leaders who evaluate and report on emerging evidence relevant to the COVID-19 pandemic, to inform Ontario's response. Our mandate is to provide weekly summaries of relevant scientific evidence for the COVID-19 Health Coordination Table of the Province of Ontario, integrating information from existing scientific tables, Ontario's universities and agencies, and the best global evidence. The Science Table summarizes its findings for the Health Coordination Table and the public in Science Briefs.

Correspondence to: Secretariat of the Ontario COVID-19 Science Advisory Table (info@covid19-sciencetable.ca)

Copyright: 2022 Ontario COVID-19 Science Advisory Table. This is an open access document distributed under the terms of the Creative Commons Attribution License, which permits unrestricted use, distribution, and reproduction in any medium, provided that the original work is properly cited.

The views and findings expressed in this Science Brief are those of the authors and do not necessarily reflect the views of all of the members of the Ontario COVID-19 Science Advisory Table, its Working Groups, and its partners.

\section{SCIENCE BRIEFS}

\section{Use of Rapid Antigen Tests during the Omicron Wave}

Peter Jüni, Sarah Baert, Antoine Corbeil, Jennie Johnstone, Samir N. Patel, Pavlos Bobos, Upton Allen, Kali A. Barrett, Lisa L. Barrett, Nicolas S. Bodmer, Karen B. Born, Laura Bourns, Gerald A. Evans, Jessica Hopkins, Douglas G. Manuel, Andrew M. Morris, Fahad Razak, Beate Sander, Michelle Science, Robert Steiner, Joshua Tepper, Nisha Thampi, Allison McGeer on behalf of the Ontario COVID-19 Science Advisory Table

\section{Key Message}

The emergence of the now provincially and globally dominant SARS-CoV-2 Omicron variant demands a reassessment of the diagnostic performance of rapid antigen tests.

Rapid antigen tests are less sensitive for the Omicron variant compared to the Delta variant in nasal samples, especially in the first 1-2 days after infection. However, rapid antigen tests can more reliably detect infectious cases of the Omicron variant in combined oral-nasal samples. Individuals can collect these samples by initially swabbing both cheeks, followed by the back of the tongue or throat, and then both nostrils.

In light of currently very high SARS-CoV-2 transmission rates in Ontario and the limited sensitivity of rapid antigen tests for the Omicron variant, a single negative rapid antigen test result cannot reliably rule out infection; a single negative test result is not conclusive and should not be used as a green light for abandoning or reducing precautions. Conversely, in this context, an individual with a positive rapid test result should be considered and managed as a case of COVID-19 and should immediately isolate; additional confirmation by polymerase chain reaction (PCR) is not necessary in most settings.

If asymptomatic testing strategies are considered, rapid antigen tests need to be performed frequently to be effective. When using 'Test-to-Stay' strategies as an alternative to large-scale isolation, asymptomatic close contacts of a positive case need to do rapid antigen testing daily. When using 'Voluntary Asymptomatic Screen Testing' strategies, asymptomatic individuals should do rapid antigen testing 3-5 times per week.

\section{Background}

Omicron has rapidly become the predominant variant of the SARS-CoV-2 virus in Ontario ${ }^{1}$ and internationally. ${ }^{2}$ Compared to other variants, Omicron is more likely to cause re-infections in previously infected individuals with variants other than Omicron, ${ }^{3}$ and breakthrough infections in vaccinated individuals. Vaccinated individuals who are more than two months past their second dose are being infected at rates similar to unvaccinated individuals. ${ }^{4}$

When the Delta variant predominated, rapid antigen tests were a potentially useful tool for detecting COVID-19 early and isolating infected people before they had a chance to spread the virus. They were also sensitive enough to rule out an infectious case with a single negative test, as discussed in a previous Science Brief. ${ }^{5}$ The emergence of Omicron demands a reassessment of the diagnostic performance of these tests. 


\section{Questions}

What is the sensitivity of rapid antigen tests to detect infectious Omicron cases?

What is the performance of rapid antigen tests for Omicron according to population case rate and sample type?

How should rapid antigen tests be used in the face of the Omicron variant?

\section{Findings}

\section{Sensitivity of Rapid Antigen Tests in Predominantly Nasal Samples of Patients with Delta and Omicron Infections}

We identified four ${ }^{6-9}$ and three ${ }^{9-11}$ studies that estimated the sensitivity of rapid antigen tests according to cycle threshold (Ct) value for the Delta and Omicron variant, respectively, using nasal or nasopharyngeal samples. One study evaluated both the Delta and Omicron variant. ${ }^{9}$ Figure 1 presents the results of random-effects metaanalyses estimating the sensitivity of rapid antigen tests in samples with Ct values of less than 25 found in PCR tests from patients with Delta (top) and Omicron (bottom) infections. As explained in the previous Science Brief, ${ }^{5} \mathrm{Ct}$ values of 25 or less are associated with high viral loads, a high probability of viable virus that can be detected in viral cultures, and a high probability of infectiousness. The pooled sensitivity of rapid antigen tests for the detection of Delta infections is $81.0 \%$. Conversely, the pooled sensitivity for the detection of Omicron infections is only $37.1 \%$. The ability of rapid antigen tests to detect a SARS-CoV-2 infection may depend on patient characteristics such as age and immune status, which were not accounted for in these analyses.

Sensitivity $(95 \% \mathrm{Cl})$

\section{Delta}

Kanjilal 2022

Konstantinus 2021

Sun 2022

Taylor 2021

Heterogeneity: $I^{2}=0.00 \%$

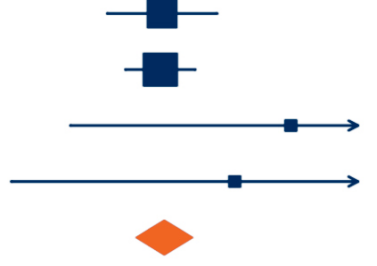

$80.0 \%$ (45.9\% to $95.0 \%$ )

$79.2 \%$ (58.7\% to $91.1 \%$ )

$99.3 \%$ (23.1\% to $100.0 \%)$

$96.9 \%$ (5.4\% to $100.0 \%)$

$81.0 \%$ (65.2\% to $90.6 \%)$

\section{Omicron}

Adamson 2022

Adamson 2022

Eckerle 2022

Kanjilal 2022

Heterogeneity: $\left.\right|^{2}=4.97 \%$

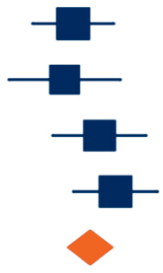

$26.7 \%(10.4 \%$ to $53.3 \%)$

$22.2 \%$ (5.6\% to $57.9 \%$ )

$43.3 \%$ (17.0\% to $74.1 \%)$

$54.5 \%$ (26.8\% to $79.7 \%$ )

$37.1 \%(23.3 \%$ to $53.0 \%)$

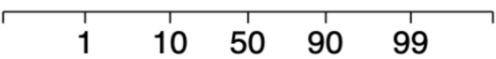

Random-effects REML model

Figure 1. Random-Effects Meta-Analysis of the Sensitivity of Rapid Antigen Tests in Nasal and Nasopharyngeal Samples with Ct Values of Less than $\mathbf{2 5}$ from Patients with Delta and Omicron Infections

Separate meta-analyses are shown for patients with Delta (top) and Omicron (bottom) infections. The two-sided $p$-value for the difference in pooled sensitivity between Delta and Omicron was $p<0.001$. Konstantinus et al ${ }^{8}$ and Eckerle et $a^{11}$ used nasopharyngeal samples, the remaining studies used nasal samples. Adamson et al ${ }^{10}$ tested two different rapid antigen tests on separate groups of samples and is included twice. Eckerle et al ${ }^{11}$ investigated five rapid antigen tests on the same samples; the sensitivity is estimated across all tests, the standard error is inflated to account for the number of samples. Kanjilal et al ${ }^{9}$ investigated both the Delta and Omicron variant and is included in both metaanalyses. Meta-analyses were done using the logits of sensitivity with a continuity correction of 0.1 in case of zero cells; estimates and 95\% Cls were then back transformed to sensitivities in the figure. $\mathrm{Cl}$, confidence interval. 
The sensitivity of rapid antigen tests for the Omicron variant may vary between different commercial tests. Figure 2 presents the sensitivity of seven different rapid antigen tests from a study in the outpatient testing centre of a hospital of symptomatic individuals infected with the Delta $(n=34)$ and Omicron $(n=36)$ variants. ${ }^{12}$ There was a significant difference in sensitivity between variants for the Panbio, Standard $Q$, Sure Status and Onsite rapid antigen tests $(p<0.001)$, but not between the remaining tests. To our knowledge, of the evaluated tests, only Panbio and Standard $Q$ are currently available in Ontario. Other rapid tests are available in Ontario but were not a part of this international study.

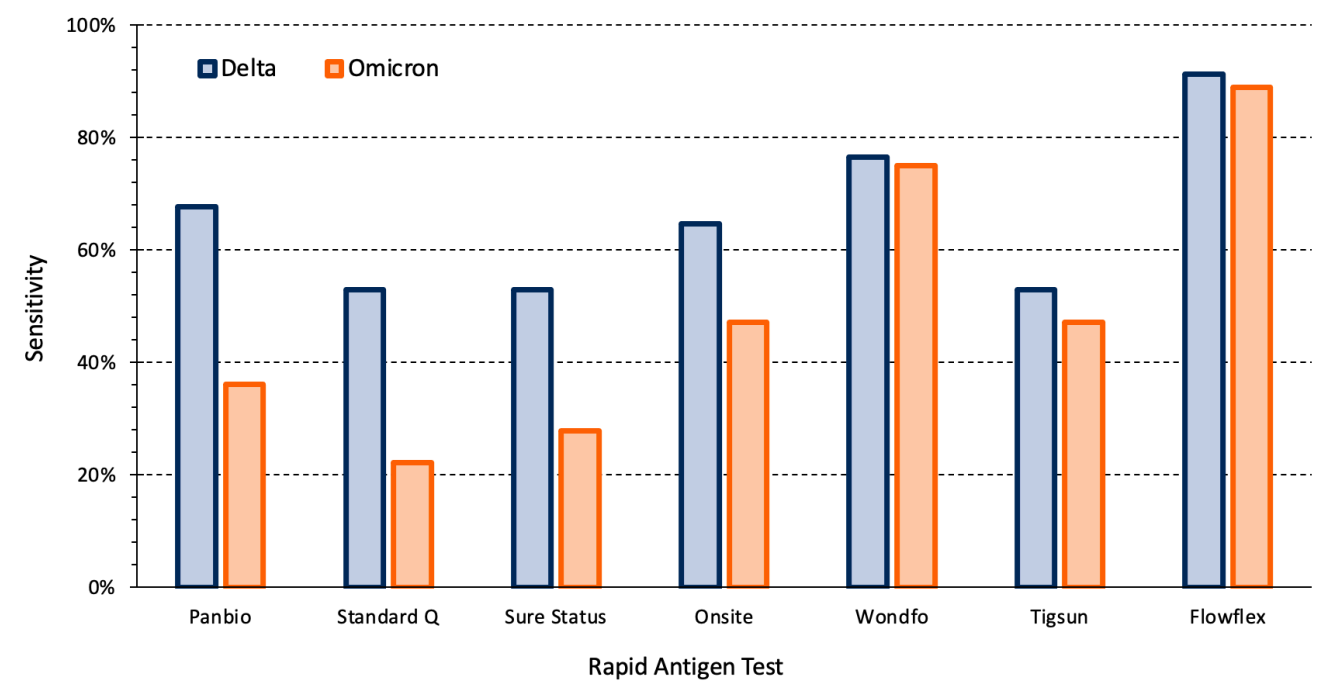

Figure 2. Sensitivity of Seven Different Rapid Antigen Tests for the Delta and Omicron Variants

Symptomatic individuals in the outpatient testing centre of a hospital infected with the Delta $(n=34)$ and Omicron ( $n=36$ ) variants were tested. Sensitivities for each variant were compared using mixed-effect logistic regression models with tests nested within patients, as two tests were performed per patient. There were significant differences in sensitivity between variants for the Panbio, Standard Q, Sure Status and Onsite rapid antigen tests ( $p<0.001)$. Viral load, presence of infectious virus and time from the first positive PCR test were comparable between Delta and Omicron groups. Data from Bekliz et al. ${ }^{12}$ PCR, polymerase chain reaction.

The sensitivity of rapid antigen tests for the Omicron variant also depends on the stage of disease progression. Figure 3 presents the change in sensitivity over time of two types of rapid antigen tests when nasal samples from a cohort of 30 individuals were tested daily during an outbreak. ${ }^{10}$ All 30 individuals developed symptoms within two days of the first positive saliva PCR test. There were no antigen positive cases until two days after the first positive saliva PCR test (Day 0), when nasal antigen test sensitivity was $16.7 \%$. Sensitivity increased to $83.3 \%$ on Day 3 and was $100 \%$ between Days 4 and 7. In a subgroup of 5 patients, viral load peaked in saliva samples 1-2 days earlier than in nasal samples. ${ }^{10}$ In summary, the majority of cases were infectious for multiple days before they had a positive rapid antigen test result. 


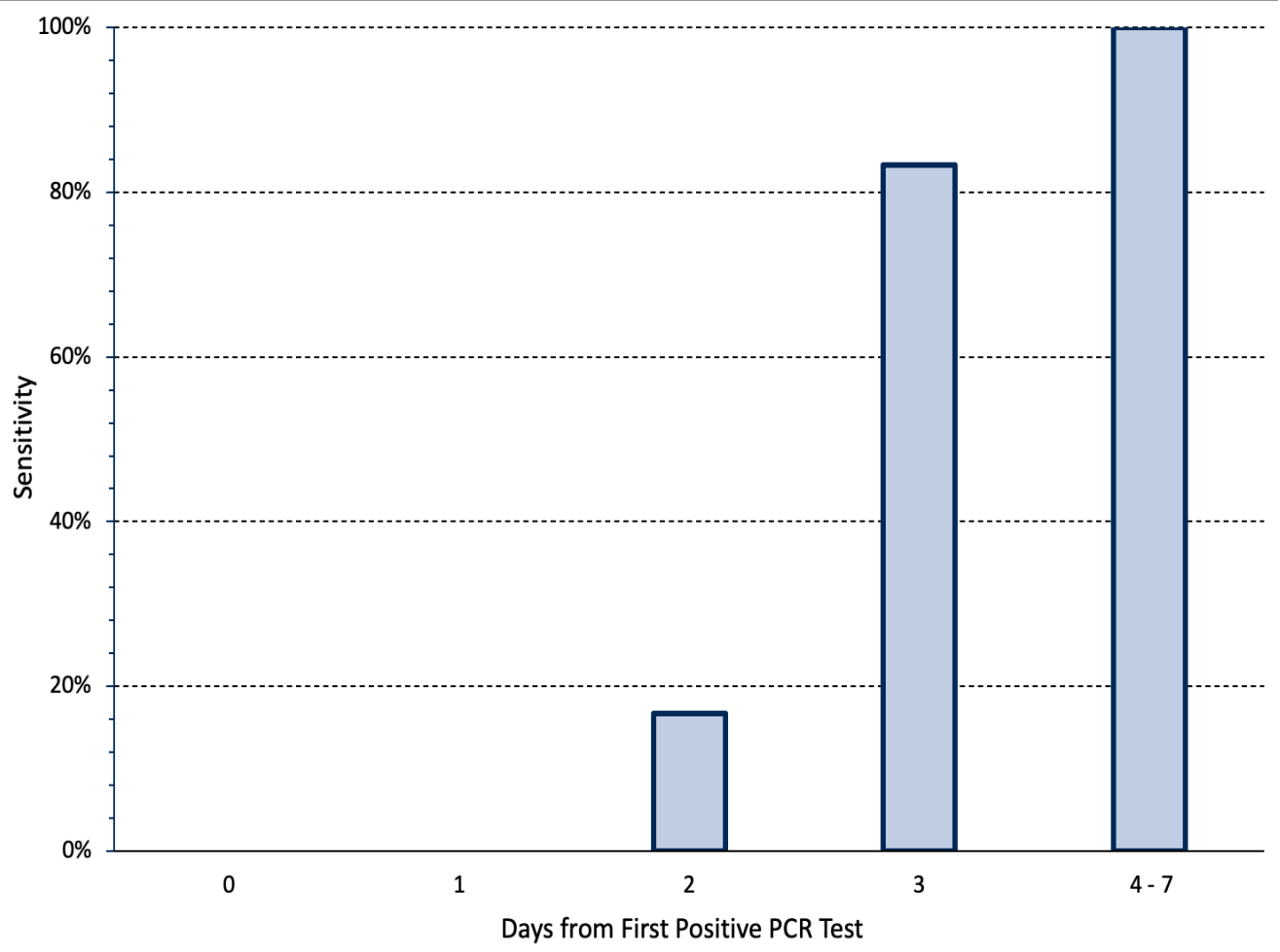

Figure 3. Temporality of Sensitivity of Daily Rapid Antigen Tests During an Omicron Outbreak

The cohort included 30 individuals in high-risk occupations who underwent daily rapid antigen testing using nasal samples after being diagnosed with COVID-19 on the basis of an initially positive saliva PCR test. All individuals had paired quantitative reverse-transcriptase $P C R$ and rapid antigen testing on day 0 or 1 relative to the first positive specimen collection date. All individuals had received two doses of a COVID-19 vaccine. Either the BinaxNOW and Quidel QuickVue rapid antigen tests were used. Day 0 was defined as time of the first positive saliva PCR test. Data from Adamson et al. ${ }^{10}$ PCR, polymerase chain reaction.

\section{Sensitivity of Rapid Antigen Tests in Nasal or Oral Samples}

The majority of the aforementioned studies used nasal sampling. Combined oralnasal sampling may improve diagnostic test sensitivity for Omicron. Figure 4 presents the relative sensitivity of PCR testing using mid-turbinate nasal and oral samples for both the Delta and Omicron variant amongst a group of acutely symptomatic, nonhospitalized individuals. ${ }^{13}$ For the Delta variant, the sensitivity of the PCR test was $100 \%$ and $71 \%$ for nasal and oral samples, respectively. For the Omicron variant, the opposite pattern was observed: sensitivity was $86 \%$ for nasal samples and $100 \%$ for oral samples.

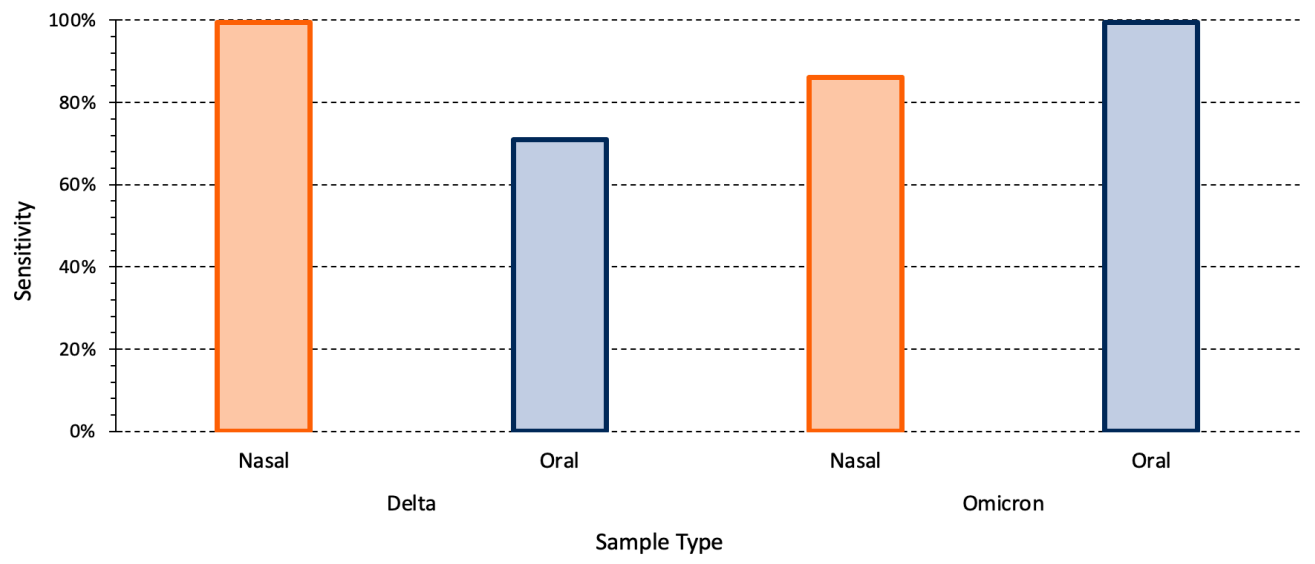

Figure 4. Sensitivity of PCR testing for the Omicron Variant in Nasal vs. Oral Samples

The relative sensitivity of mid-turbinate nasal and oral swabs (cheek, above and below the tongue, the gums and hard palate) for both the Delta and Omicron variant amongst a group of acutely symptomatic, non-hospitalized individuals (22 individuals with Delta infection, 31 individuals with Omicron infection). Data from Marais et al. ${ }^{13}$

Combining oral and nasal sampling may improve the sensitivity of rapid antigen 
tests. Figure 5 compares the sensitivity of the Abbott Panbio rapid antigen test for the Omicron variant using a nasal sample alone versus a combined throat and nasal sample in a study of 520 individuals, 38 of whom were confirmed positive by PCR. ${ }^{14}$ The sensitivity of a nasal sample alone was $68.4 \%$, while the sensitivity of a combined nasal and throat sample was $81.6 \%$. Additional analyses combining results from nasal samples alone and throat samples alone to approximate the sensitivity of a combined sample demonstrated similar results, but with narrower $95 \%$ confidence intervals. ${ }^{14}$

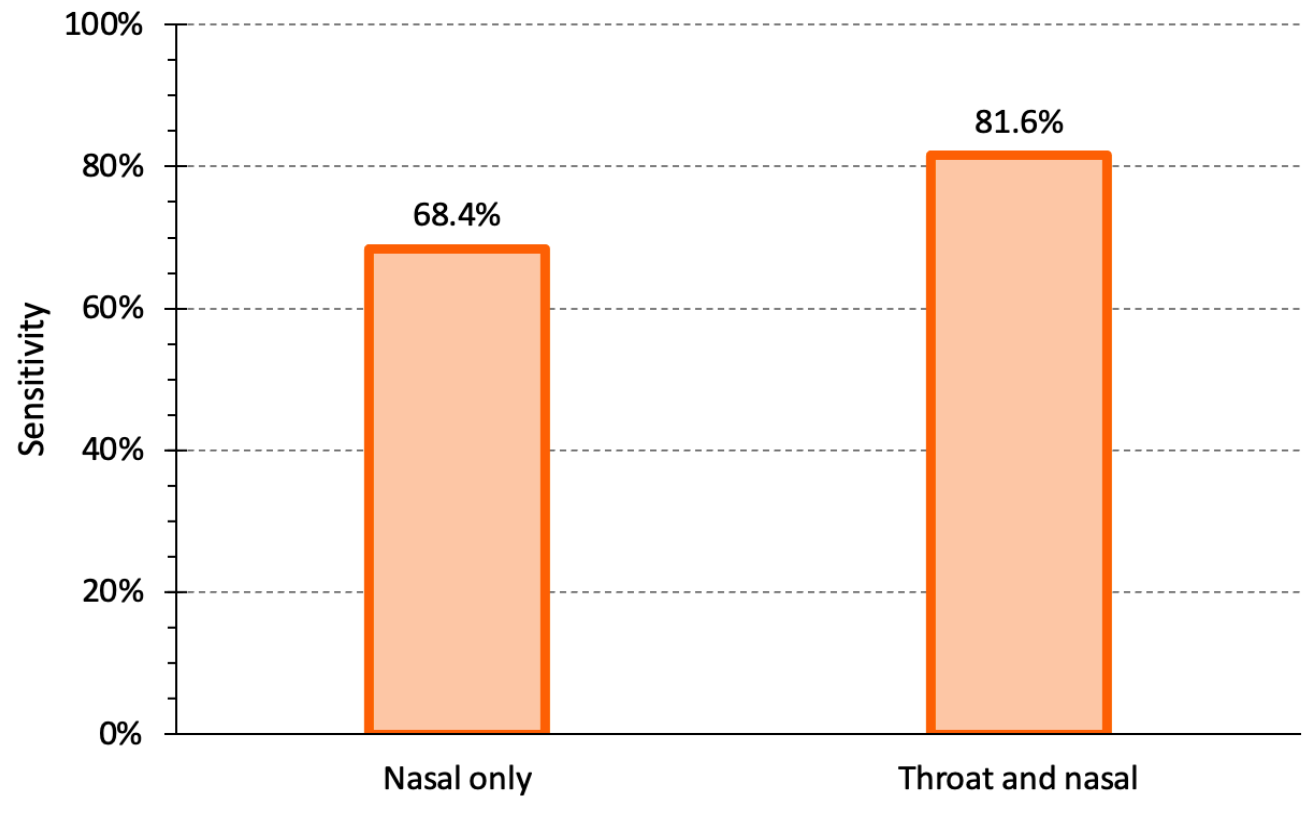

Figure 5. Sensitivity of the Abbott Panbio Rapid Antigen Testing for the Omicron Variant in Nasal versus Combined Throat and Nasal Samples

The sensitivity of the Abbott Panbio rapid antigen test for the Omicron variant using a nasal sample alone vs. a combined throat and nasal sample. There were 520 individuals total, 38 of whom were positive. The sensitivity of nasal sample alone was $68.4 \%$ (95\% confidence interval (CI) 51.4 to $82.5 \%$ ), while the sensitivity of a combined nasal and throat sample was $81.6 \%$ (95\% Cl 65.7 to 92.3\%). The specificity of Abbott Panbio was $100 \%$ (95\% Cl 99.2 to $100 \%$ ). Note that the sensitivity for nasal swabs in this study is higher than the pooled sensitivity seen for nasal/ nasopharyngeal swabs of infectious Omicron cases in samples with Ct values of less than 25 in our meta-analysis (see Figure 1). Data from Goodall et al. ${ }^{14}$

Self-collected combined oral and nasal samples have been previously validated in Ontario as a method for detecting SARS-CoV-2 via PCR; ${ }^{15}$ in this case, the oral swabs were from the buccal (cheek) and tongue surfaces. In addition, combined throat and nasal samples have been previously shown to have comparable sensitivity to nasopharyngeal samples. ${ }^{16,17}$

Although there is no direct evidence, the above studies suggest an approach of combining oral and nasal samples, beginning by swabbing both cheeks and the back of the tongue or throat, before swabbing both nostrils. Swabbing the back of the tongue or throat may induce a gag reflex, which is not dangerous and actually indicates that the location of the swabbing was appropriate. Video instructions on how to perform a combined oral-nasal swab can be found here for adults and here for children. This method will differ from test manufacturer instructions that describe nasal or nasopharyngeal sample collection; however, a combined oral-nasal swab can be used for any rapid antigen test, irrespective of the originally intended sample type.

\section{Ability of Rapid Antigen Tests to Detect or Rule Out Infectious Cases}

We have established that the sensitivity of rapid antigen tests is lower for the Omicron variant than for the Delta variant, particularly in the first few days of infection, and that nasal samples are less sensitive than combined oral-nasal samples. We now return to the patient themselves to assess the probability of being an infectious case after a positive or negative rapid antigen test, taking the rates of reported cases in Ontario 
or a Public Health Unit after the change in testing strategy in December 2021 into account. In contrast to previous variants, unvaccinated people and vaccinated people have similar pre-test probabilities of disease. ${ }^{4}$ Consequently, unvaccinated people and vaccinated people also have similar post-test probabilities of disease, if vaccinated individuals have only received 1 or 2 doses, and the second dose was administered more than 2 months ago.

Figure 6 presents the relationship between the daily rate of reported COVID-19 cases in Ontario (after the change in testing strategy) and the post-test probability of an infectious Omicron case after a positive or negative rapid antigen test. The posttest probability is influenced by the pre-test probability, the test result (positive vs. negative), and by the positive and negative likelihood ratios of the rapid antigen test being used.

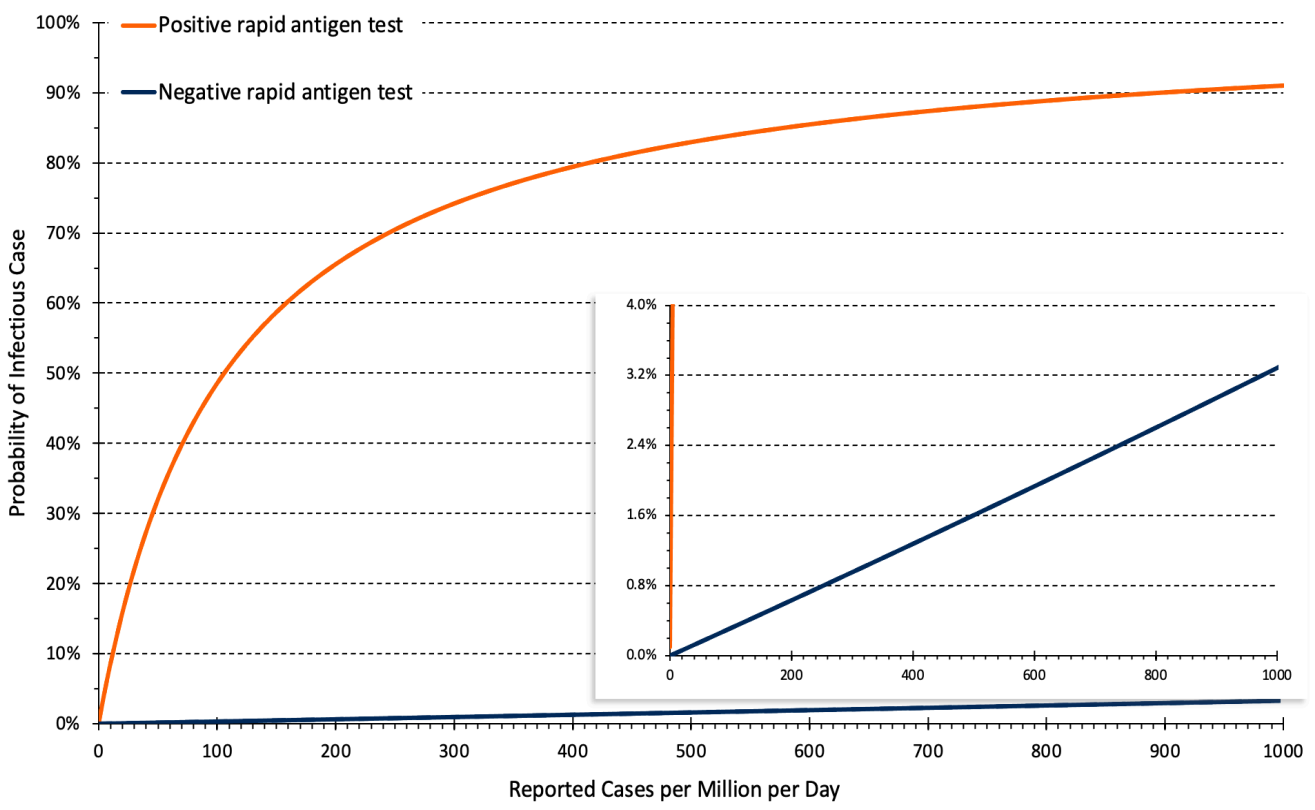

Figure 6. Estimated Probability of Infectious Case after a Positive or Negative Rapid Antigen Test with Combined Oral and Nasal Samples

Relationship between the daily rate of COVID-19 cases in Ontario and post-test probability of an infectious case after a positive and negative rapid antigen test. Estimates are based on 14-day averages of the overall reported case rate after the change in testing strategy at the end of December 2021. The rate of cases per million per day needs to be multiplied by 0.7 to derive a rate of cases per 100,000 per week, e.g., 50 reported cases per million per day correspond to 35 cases per 100,000 per week. Based on triangulations using wastewater data ${ }^{1}$ and reported cases, ${ }^{1}$ we assumed that the altered testing strategy detects approximately $10 \%$ of infections. Pre-test probabilities of infection were assumed to be similar for unvaccinated and vaccinated individuals if vaccination constituted 1 or 2 doses, and the second dose was administered more than 2 months ago. ${ }^{4}$

In light of the results of our meta-analysis (Figure 1) and the likely increase in sensitivity of rapid antigen tests to detect infectious Omicron cases when using a combined oral-nasal sample, we assume a sensitivity of $60 \%$ for rapid antigen tests when using a combined sample, and a specificity of $99.5 \% .{ }^{18-20}$ We therefore estimate a positive likelihood ratio of $0.60 /(1-0.995)=120$, indicating that a positive test is highly predictive of an infectious case when using a combined oral-nasal sample.

By contrast, the estimated negative likelihood ratio is $(1-0.60) / 0.995=0.40$, indicating that negative rapid antigen tests have only limited power to rule out an infectious case, particularly in the first 1-2 days of infection (Figure 3 ). This limitation is most salient when the pre-test probability of disease is high, as is currently the case during the Omicron wave.

On February 1, 2022, the approximate rate of reported COVID-19 cases in Ontario was 260 per 1 million inhabitants per day (180 per 100,000 per week). Assuming that approximately $10 \%$ of infections are reported with the current testing strategy, this number corresponds to a true case rate of 2,600 infections per million per day (1800 
per 100,000 per week). At this rate, the estimated pre-test probability of an infectious case in the general population of Ontario is about $2 \%$. After a positive rapid antigen test, the probability of an infectious case increases from $2 \%$ to $70 \%$ (Figure 6).

By contrast, after a negative rapid antigen test, the probability of an infectious case only decreases from $2 \%$ to $0.8 \%$ (Figure 6 ). Rapid antigen tests, when negative, will therefore need to be repeated frequently to be clinically relevant.

Changes in the population case rate are relevant to future decisions about the use of rapid antigen tests. If, with the current testing strategy, the case rate falls to 50 reported cases per 1 million inhabitants per day (35 per 100,000 per week), the approximate pre-test probability of disease will be only $0.4 \%$. In this scenario, the reduction in probability afforded by a single negative rapid antigen test is only $0.2 \%$, from $0.4 \%$ to $0.2 \%$. This decrease continues to be clinically irrelevant. The approach with respect to positive rapid antigen tests may change however, as the probability of an infectious case after a positive test is now only $33 \%$. As such, 2 out of 3 positive rapid antigen tests could be false positives, and a positive result should be confirmed by a PCR test at this case rate.

\section{Frequency of Testing}

The results of negative rapid antigen tests are more reliable when they are used serially. Figure 7 presents an epidemiological scenario modelled by Paltiel et $a^{21}$ for unvaccinated college students that is similar to the Omicron wave in Ontario in early February 2022 and demonstrates the importance of testing frequency in reducing transmission.

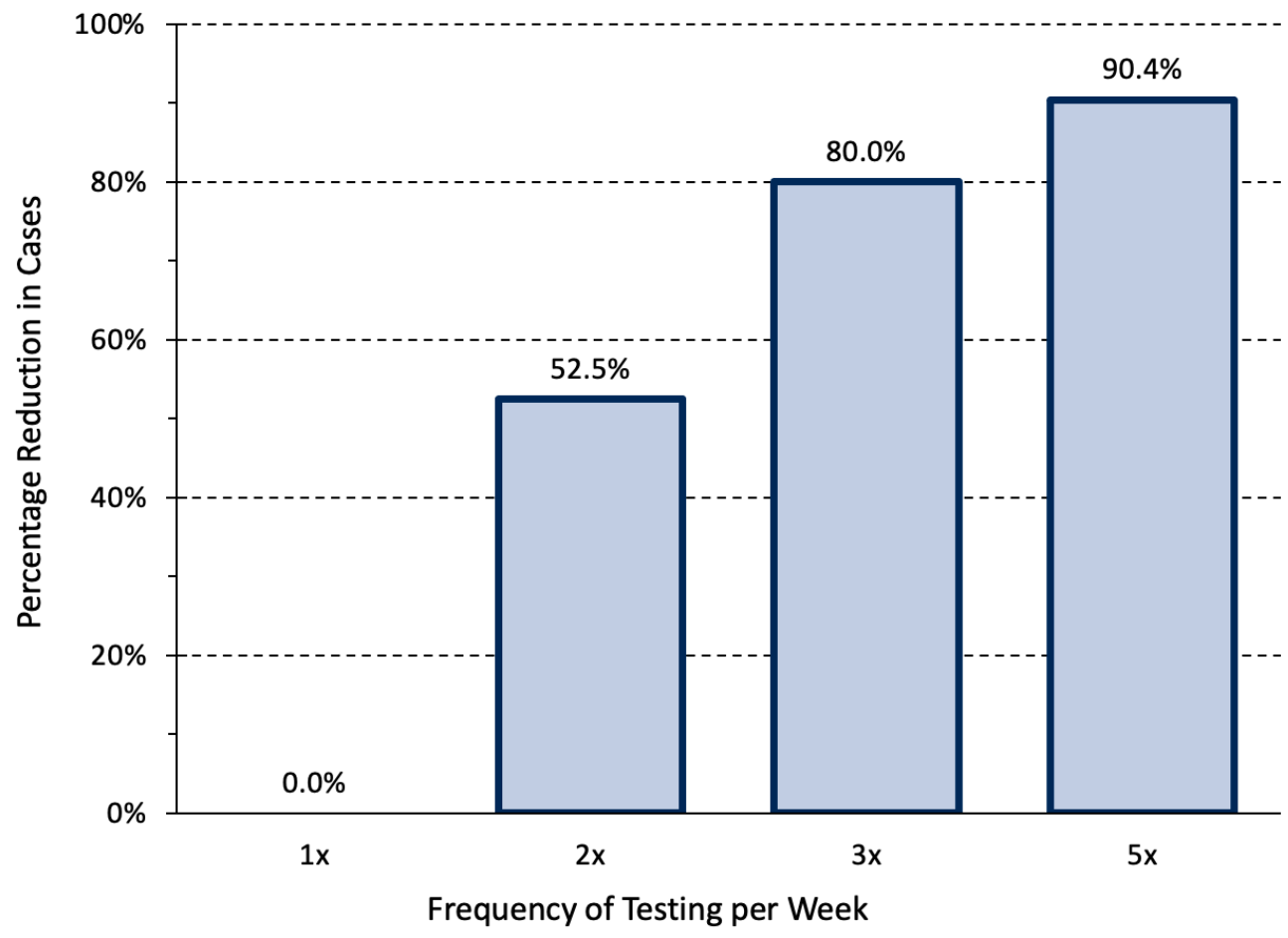

Figure 7. Relative Reduction in COVID-19 Cases Afforded by Different Frequencies of Rapid Antigen Testing Relative reduction in COVID-19 cases afforded by different frequencies of rapid antigen testing. Modelling was based on a hypothetical cohort of 5000 unvaccinated college students followed for 80 days when the wild type virus was dominant. The modelled scenario is closest to Ontario's current situation: it assumes a lower initial rate (700 infections per 1 million inhabitants per day, 490 per 100,000 per week), but strong exponential growth. Assuming that approximately $10 \%$ of infections are reported with the current testing strategy, this scenario would correspond to 70 reported COVID-19 cases per 1 million inhabitants per day (49 per 100,000 per week) in Ontario. At a test sensitivity of $70 \%$, weekly testing is insufficient and has no impact on transmission. In comparable situations, rapid antigen testing with combined oral-nasal samples should therefore be performed 3 to 5 times per week. Data from Paltiel et al. ${ }^{21}$

At an assumed test sensitivity of $70 \%$, weekly testing was insufficient to reduce transmission. If rapid antigen tests are employed for voluntary screen testing in 
Ontario where the current pre-test probability of disease is high, combined oral-nasal samples should be used, and testing should be performed 3 to 5 times per week in order to meaningfully reduce transmission.

\section{Interpretation}

Combined oral-nasal sampling that involves swabbing both cheeks, the back of the tongue or throat, then both nostrils, should be implemented for rapid antigen tests. In light of the high pre-test probability of disease in Ontario and the limited sensitivity of rapid antigen tests for the Omicron variant, a single negative rapid antigen test result should not be used to rule out a case of COVID-19 at this time. Expressed differently, a negative test result is not conclusive and is not a green light for abandoning precautions. However, at the time of writing, a single positive rapid test result can be used to confirm COVID-19. We therefore advise the following for testing symptomatic individuals:

\section{Confirming COVID-19 in the Case of Symptoms}

If someone is symptomatic, they are presumed to have COVID-19 and must isolate as soon as possible regardless of rapid antigen test results. If a rapid antigen test is done and is positive, it should be treated as diagnostic for COVID-19. Additional confirmation by PCR is not necessary in most situations. Following a return to work, individuals should wear properly fitted, high-quality masks and practice physical distancing whenever possible.

With the current testing strategy, once the reported rate falls below 50 reported cases per 1 million inhabitants per day (35 per 100,000 per week), 2 out of 3 positive rapid antigen tests could be a false positive. At this point, confirmation of all positive rapid antigen test results by a PCR test should be considered for the general public, and general recommendations on the use of rapid antigen tests should be re-evaluated.

COVID-19 treatment decisions should not be based solely on a positive rapid antigen test. Treatment may be initiated based on a combination of clinical presentation and a positive rapid antigen test, but confirmation by a rapid molecular test or PCR test should be sought in addition.

\section{Ruling Out COVID-19 in the Case of Symptoms}

If rapid antigen tests are available to a person with symptoms of COVID-19, they may be used to assess the likelihood that symptoms are due to infection with SARS-CoV-2. A single negative test in an individual with compatible symptoms does not mean that they do not have COVID-19 infection. There are multiple ways in which serial testing can be applied to assess the likelihood of COVID-19, depending on the case rate, risk tolerance, and setting. Options include two consecutive negative tests, separated by 24-48 hours, as is the current provincial guidance, ${ }^{22}$ or two consecutive negative tests, spaced 3-5 days apart, as suggested by Figure 3. Regardless of the serial testing strategy, current provincial guidance ${ }^{22}$ states that individuals should self-isolate until they have no fever, and symptoms are improving for at least 24 hours (or 48 hours if gastrointestinal symptoms).

If rapid antigen tests are used for asymptomatic screen testing, performing them frequently will maximize their value. We therefore advise the following if testing of asymptomatic individuals, outside of high-risk settings, is considered:

If Rapid Antigen Testing Is Used as an Alternative to Large-Scale Isolation When a New Case Is Detected

This approach is called "Test-to-Stay," and is used in moderate-risk settings for SARSCoV-2 transmission where individuals congregate outside of their household (such 
as schools and workplaces). Individuals who have had contact with the positive individual are tested daily with rapid antigen tests for the period of risk (i.e., 5-10 days after contact with the person who tested positive). If individuals continue to produce daily negative results and do not show symptoms, they can remain on-site rather than isolating at home, provided that they properly mask and practice physical distancing whenever possible. Current provincial guidance also provides variations of this approach for different levels of critical staffing shortages..$^{23}$ It is important to note that case and contact management leading to large-scale isolation is not currently the norm in moderate-risk settings in Ontario.

\section{If Voluntary Asymptomatic Screen Testing Is Used}

This approach entails regular voluntary rapid antigen testing of asymptomatic individuals to find cases in moderate-risk settings for SARS-CoV-2 transmission, where individuals congregate outside of their household (such as schools and workplaces). In order to meaningfully reduce transmission in these settings, asymptomatic individuals would need to perform rapid antigen testing 3-5 times per week. With the current testing strategy, if the case rate falls to 20 reported cases per 1 million inhabitants per day (14 per 100,000 per week), and there is no evidence of exponential growth, voluntary asymptomatic screen testing is unlikely to meaningfully reduce infections.

High-risk settings for SARS-CoV-2 transmission (e.g., acute care, long-term care, prisons) have distinct strategies for asymptomatic testing and are not covered in this Science Brief. Studies of analytical sensitivity have not been considered here given important limitations, particularly the potential decoupling of viral load (or Ct value) with infectiousness in vivo. ${ }^{24,25}$

While rapid tests may help with detection of infectious cases, they are likely insufficient for controlling spread. Successful implementation of a rapid testing program relies on ensuring that supports are in place for all related activities, including distribution, case and contact management, lab-based testing (depending on the case rate), and education. Additional implementation challenges include the current global supply shortage of rapid antigen tests, which may prevent the high frequency testing strategies described above.

\section{Methods Used for This Science Brief}

We searched PubMed, Google Scholar, the COVID-19 Rapid Evidence Reviews, the Joanna Briggs Institute's COVID-19 Special Collection, LitCovid in PubMed, the Oxford COVID-19 Evidence Service, the World Health Organization's Global Literature on Coronavirus Disease, and other COVID-19 specific resources listed by the McMaster Health Forum. In addition, we retrieved reports citing relevant articles through Google Scholar and reviewed references from identified articles for additional studies. The search was last updated on February 2, 2022.

We performed a random-effects meta-analysis of the sensitivity of rapid antigen tests in samples with Ct values of less than 25 from patients with Delta and Omicron infections. Meta-analyses were done using the logits of sensitivity with a continuity correction of 0.1 in case of zero cells; estimates and $95 \% \mathrm{Cls}$ were then back transformed to sensitivities.

The relationship between the rate of cases per million inhabitants per day and the post-test probability after a positive or negative rapid antigen test was estimated based on pre-test probabilities, which were a function of rates of diagnosed cases. We transformed these probabilities into pre-test odds, multiplied the pre-test odds with likelihood ratios to derive post-test odds, and back-transformed these post-test odds into post-test probabilities. To derive pre-test probabilities we assumed that $10 \%$ of actual SARS-CoV-2 infections were diagnosed in Ontario after the change of the 
testing strategy in late December 2021.

\section{Author Contributions}

PJ conceived the Science Brief. PJ and PB performed analyses. PJ and SB performed literature searches and wrote the first draft of the Science Brief. PJ and PB extracted data. All authors revised the Science Brief critically for important intellectual content and approved the final version.

\section{References}

1. Jüni P, da Costa BR, Maltsev A, et al. Ontario Dashboard. Sci Briefs Ont COVID-19 Sci Advis Table. Published online January 1, 2021. https://doi.org/10.47326/ocsat. dashboard.2021.1.0

2. Hodcroft EB. CoVariants: SARS-CoV-2 mutations and variants of interest. Published 2022. Accessed January 30, 2022. https://covariants.org/

3. Pulliam JRC, Schalkwyk C van, Govender N, et al. Increased risk of SARSCoV-2 reinfection associated with emergence of the Omicron variant in South Africa. medRxiv; 2021:2021.11.11.21266068. https://doi. org/10.1101/2021.11.11.21266068

4. Buchan SA, Chung H, Brown KA, et al. Effectiveness of COVID-19 vaccines against Omicron or Delta symptomatic infection and severe outcomes. Published online January 28, 2022. medRxiv; https://doi.org/10.1101/2021.12.30.21268565

5. Jüni $P$, Baert $S$, Bobos $P$, et al. Rapid antigen tests for voluntary screen testing. Sci Briefs Ont COVID-19 Sci Advis Table. 2021;2(52). https://doi.org/10.47326/ ocsat.2021.02.52.1.0

6. Sun KJ, Vaeth MJE, Robinson M, et al. High sensitivity and NPV for BinaxNOW rapid antigen test in children at a mass testing site during prevalent Delta variant. medRxiv; 2022:2022.01.05.22268788. https://doi.org/10.1101/2022.01.05.22268788

7. Taylor A, Calvez R, Atkins M, Fink CG. Comparing lateral flow testing with a rapid RT-PCR method for SARS-CoV-2 detection in the UK. medRxiv; 2021:2021.10.08.21264742. https://doi.org/10.1101/2021.10.08.21264742

8. Konstantinus I, Chiwara D, Ndevaetela EE, et al. Laboratory and field evaluation of the STANDARD Q and PanbioTM SARS-CoV-2 antigen rapid test in Namibia using nasopharyngeal samples. medRxiv; 2021:2021.09.21.21263886. https://doi. org/10.1101/2021.09.21.21263886

9. Kanjilal S, Chalise $S$, Shah AS, et al. Analytic sensitivity of the Abbott BinaxNOWTM lateral flow immunochromatographic assay for the SARSCoV-2 Omicron variant. medRxiv; 2022:2022.01.10.22269033. https://doi. org/10.1101/2022.01.10.22269033

10. Adamson B, Sikka R, Wyllie AL, Premsrirut P. Discordant SARS-CoV-2 PCR and rapid antigen test results when infectious: A December 2021 occupational case series. medRxiv; 2022:2022.01.04.22268770. https://doi. org/10.1101/2022.01.04.22268770

11. Eckerle I. Retrospective testing of nasopharyngeal patient samples. @ Eckerlelsabella. Published December 30, 2021. Accessed January 10, 2022. https://twitter.com/Eckerlelsabella/status/1476503366901673986

12. Bekliz M, Perez-Rodriguez F, Puhach O, et al. Sensitivity of SARS-CoV-2 antigendetecting rapid tests for Omicron variant. Published online January 17, 2022. medRxiv; https://doi.org/10.1101/2021.12.18.21268018 
13. Marais G, Hsiao N yuan, Iranzadeh A, et al. Saliva swabs are the preferred sample for Omicron detection. medRxiv; 2021:2021.12.22.21268246. https://doi. org/10.1101/2021.12.22.21268246

14. Goodall BL, LeBlanc JJ, Hatchette TF, Barrett L, Patriquin G. Investigating sensitivity of nasal or throat (ISNOT): A combination of both swabs increases sensitivity of SARS-CoV-2 rapid antigen tests. Published online January 21, 2022. medRxiv; https://doi.org/10.1101/2022.01.18.22269426

15. Kandel CE, Young M, Serbanescu MA, et al. Detection of severe acute respiratory coronavirus virus 2 (SARS-CoV-2) in outpatients: A multicenter comparison of self-collected saline gargle, oral swab, and combined oral-anterior nasal swab to a provider collected nasopharyngeal swab. Infect Control Hosp Epidemiol. 2021;42(11):1340-1344. https://doi.org/10.1017/ice.2021.2

16. Tsang NNY, So HC, Ip DKM. Is oropharyngeal sampling a reliable test to detect SARS-CoV-2? Lancet Infect Dis. 2021;21(10):1348-1349. https://doi.org/10.1016/ S1473-3099(21)00402-3

17. Tsang NNY, So HC, Ng KY, Cowling BJ, Leung GM, Ip DKM. Diagnostic performance of different sampling approaches for SARS-CoV-2 RT-PCR testing: A systematic review and meta-analysis. Lancet Infect Dis. 2021;21(9):1233-1245. https://doi. org/10.1016/S1473-3099(21)00146-8

18. Gans JS, Goldfarb A, Agrawal AK, Sennik S, Stein J, Rosella L. False-positive results in rapid antigen tests for SARS-CoV-2. JAMA. 2022;327(5):485-486. https://doi. org/10.1001/jama.2021.24355

19. Hledík M, Polechová J, Beiglböck M, Herdina AN, Strassl R, Posch M. Analysis of the specificity of a COVID-19 antigen test in the Slovak mass testing program. PLoS ONE. 2021;16(7):e0255267. https://doi.org/10.1371/journal.pone.0255267

20. Institute of Population Health, University of Liverpool. Covid-SMART asymptomatic testing pilot in Liverpool city region: Quantitative evaluation. Accessed February 9, 2022. https://www.liverpool.ac.uk/media/livacuk/coronavirus/Liverpool_City_ Region_Covid_SMART_Evaluation.pdf

21. Paltiel AD, Zheng A, Walensky RP. Assessment of SARS-CoV-2 screening strategies to permit the safe reopening of college campuses in the United States. JAMA Netw Open. 2020;3(7):e2016818. https://doi.org/10.1001/ jamanetworkopen.2020.16818

22. Ontario Ministry of Health. COVID-19 Integrated testing \& case, contact and outbreak management interim guidance: Omicron surge.; 2022:20. https://www. health.gov.on.ca/en/pro/programs/publichealth/coronavirus/docs/contact_ mngmt/management_cases_contacts_omicron.pdf

23. Ontario Ministry of Health. COVID-19 interim guidance: Omicron surge management of critical staffing shortages in highest risk settings.; 2022:6. https:// www.health.gov.on.ca/en/pro/programs/publichealth/coronavirus/docs/early return\%20_to_work.pdf

24. Hay JA, Kissler SM, Fauver JR, et al. Viral dynamics and duration of PCR positivity of the SARS-CoV-2 Omicron variant. medRxiv; 2022:2022.01.13.22269257. https:// doi.org/10.1101/2022.01.13.22269257

25. Puhach O, Adea K, Hulo N, et al. Infectious viral load in unvaccinated and vaccinated patients infected with SARS-CoV-2 WT, Delta and Omicron. medRxiv; 2022:2022.01.10.22269010. https://doi.org/10.1101/2022.01.10.22269010 